\title{
ILCEA
}

Revue de l'Institut des langues et cultures

d'Europe, Amérique, Afrique, Asie et Australie

$16 \mid 2012$

La culture progressiste à l'époque de la guerre froide

\section{La musique électroacoustique en République démocratique allemande (RDA) : une avant-garde paradoxale}

\section{Tatjana Böhme-Mehner}

\section{(2) OpenEdition}

\section{Journals}

Édition électronique

URL : http://journals.openedition.org/ilcea/1445

DOI : 10.4000/ilcea.1445

ISSN : 2101-0609

Éditeur

UGA Éditions/Université Grenoble Alpes

Édition imprimée

ISBN : 978-2-84310-232-5

ISSN : 1639-6073

Référence électronique

Tatjana Böhme-Mehner, « La musique électroacoustique en République démocratique allemande (RDA) : une avant-garde paradoxale», ILCEA [En ligne], 16 | 2012, mis en ligne le 04 juillet 2012, consulté le 22 mars 2021. URL : http://journals.openedition.org/ilcea/1445 ; DOI : https://doi.org/ 10.4000/ilcea.1445

Ce document a été généré automatiquement le 22 mars 2021.

(c) ILCEA 


\title{
La musique électroacoustique en République démocratique allemande (RDA) : une avant-garde paradoxale
}

\author{
Tatjana Böhme-Mehner
}

\section{Introduction - Peut-on parler d'une musique électroacoustique est-allemande?}

1 En écoutant des productions musicales réalisées au « Labor für akustisch-musikalische Grenzprobleme " (laboratoire examinant les problèmes à l'interface acoustique/ musique) pour la radio est-allemande des années 1950 et 1960, l'auditeur est inévitablement surpris. Il ne s'agit pas de ce que l'on associe généralement à l'expression "musique moderne». Il paraît assez difficile de classifier les sons entendus, tant au niveau historique qu'esthétique. L'analyse critique du style, comme méthode centrale de la musicologie, ne guide à rien d'essentiel.

2 On peut trouver un air postromantique dans cette musique, en même temps qu'une certaine attitude modérée ou de distraction. Mais ces moments n'apparaissent ni comme vraiment caractéristiques, ni comme tout à fait originaux. Quelques instants plus tard, une attitude Stravinskienne émane du complexe. La pensée orchestrale reste dominante. D'un autre côté, il y a aussi des sons étranges dans cette pièce. Ces sons n'apparaissent pas comme véritablement modernes, encore moins de caractère naturel. Ce sont donc des sons synthétiques, qui ne sont pourtant pas tellement éloignés d'une pensée harmonique traditionnelle. 


\section{L'histoire étrange du « Labor für akustisch- musikalische Grenzprobleme »}

3 Si l'on prend la Rhapsodie de Hans-Hendrik Wehding, et quand on réfléchit qu'il s'agit d'une composition produite au milieu des années 1960, il est clair que cette musique ne pouvait pas - à l'époque de sa production - être avant-gardiste, pas même moderne. À l'époque, Stockhausen avait déjà terminé sa période radicalement électronique. On avait, à l'Est de l'Allemagne, Eisler et Dessau. C'était l'époque des grands succès des Beatles. On pourrait continuer avec une longue liste des contraires et contrastes représentant les visages différents du progressisme à l'Est comme à l'Ouest de l'Europe.

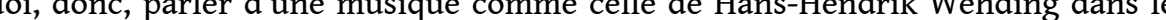
cadre d'un colloque sur la culture progressiste? Première réponse: parce qu'elle représente un aspect d'un phénomène que nous appelons l'Avant-gardisme-Paradoxe de la musique électroacoustique en RDA. Nous sommes malgré tout convaincue que l'analyse des relations dans ce contexte peut permettre la compréhension des interpénétrations du progressisme social, culturel, technologique, esthétique et du conservatisme dans les domaines mentionnés.

5 La musique de Wehding représente un cas particulier de ce phénomène est-allemand, et elle est typique d'un certain monde sonore de l'Est, qui est mal connu, même dans son pays d'origine.

6 Aussi, si l'on suit les catégories et mesures esthétiques du réalisme socialiste, cette musique ne peut pas être considérée comme conforme au règlement du système. Elle peut être classifiée comme bourgeoise d'une certaine manière, voire réactionnaire. Mais, malgré tout, elle représente le progressisme en soi sur le plan technologique. C'est ce que les techniciens et ingénieurs au «Labor für akustisch-musikalische Grenzprobleme » à Berlin ont cru, et du point de vue techno-historique ce n'est pas faux du tout. La composition de Wehding a été produite dans le premier studio électronique du bloc de pays de l'Est, avec une quarantaine d'autres compositeurs.

7 Le premier studio électronique du bloc de pays de l'Est était à Berlin - cela peut être reçu comme choquant, dérangeant, en tout cas comme non réellement conforme aux représentations historiographiques de la RDA et de la musique électroacoustique. Fait surprenant, même pour une grande partie des musicologues historiens, le «Labor für akustisch-musikalische Grenzprobleme" était non seulement le premier studio électronique à l'Est de l'Europe, mais fut aussi le premier à être fermé. L'expérience de la production de la musique électroacoustique était déclarée comme ratée. Pour résumer les données historiographiques de cette institution en quelques lignes, soulignons qu'elle fut fondée en 1956, d'abord comme organisme de recherche de base et appliquée à la radio au niveau technologique. En incitant, au sein d'un laboratoire, le développement d'une technologie adaptée aux médias contemporains, on incluait dans les tâches de l'unité le développement d'une sonorité nouvelle et d'appareils utilisables pour la production d'une musique électroacoustique suivant une esthétique particulière.

8 Un des objectifs centraux était donc la réalisation d'un instrument électronique, le "Subharchord", jusqu'à sa production en série. Les constructeurs se basèrent sur la tradition du "Trautonium », construit par Friedrich Trautwein et développé plus tard par Oskar Sala, qui le dote d'un clavier traditionnel (de piano). Ils prirent en compte, 
pour leur construction, la théorie des sous-harmonies, généralement assez controversée, qui cherche une échelle de sous-harmonies en tant qu'un pendant de l'échelle des harmoniques supérieures. En général, le laboratoire se réfère à l'histoire de l'institut de l'entre-deux-guerres «Rundfunkversuchsstelle» (centre d'essai radiophonique) à Berlin-Charlottenburg. Ce centre était perçu à la fois comme progressiste et de tradition humaniste - notamment à cause de sa fermeture par les Nazis.

9 En 1970, le laboratoire arrête pourtant ses travaux autour de l'art sonore, et l'équipe est associée à un département de recherche plus large, celui de la " poste » (le département technologique de la radio). L'ouverture prévue d'un vrai studio (artistique) d'art sonore est annulée. Le résultat de nos recherches dans des archives privées et publiques concernant l'histoire du studio, montre clairement que l'arrêt des travaux n'était pas lié en premier lieu à des pressions esthétiques ou politiques, mais économiques. L'instrument ne se vendait pas autant que prévu au niveau international. Même si l'intérêt que nombreux studios et instituts internationaux lui portaient est documenté, notamment concernant la technologie de filtrage, ils l'estimaient trop cher.

10 L'abandon des travaux à Adlershof ne fut presque pas remarqué par le grand public. Le progressisme esthétique avait déjà pris d'autres directions.

\section{Impressions par-delà l'officiel}

11 Lothar Voigtländer gagnait avec son œuvre Maikäfer flieg de 1985 le premier prix au festival de Bourges, ce qui lui valut sans doute la valeur esthétique d'être avantgardiste. Il l'était certainement, mais il était aussi engagé politiquement et, à un certain niveau, opposant du régime. Voigtländer emploie des sons concrets, pris de contextes divers, bien que souvent militaires, qui se différencient des anciennes marches militaires, ou même militaristes, par les bruits d'un hélicoptère. Après un passage extrêmement calme, l'œuvre arrive à son point culminant : une voix fragile chante la chanson enfantine Maikäfer flieg. La phrase « Mutter ist in Pommerland, Pommerland ist abgebrandt " était mise à l'index à l'époque parce qu'elle touchait le sujet, également proscrit, des anciens territoires allemands à l'Est. C'était, bien sûr, provocateur.

12 Sur le plan technologique pourtant, c'est un peu bricolé, improvisé, on est loin du dernier cri ou du progressisme pur et dur...

13 Même si notre intention ne peut certainement pas être ici de mettre en relation le progressisme esthétique et technologique de niveaux différents, il est malgré tout important de souligner que Voigtländer n'a pas pu produire dans un studio public et parfaitement équipé dans son pays d'origine, parce qu'à l'époque il n'en existait aucun.

14 À peu près au moment où le studio à Adlershof fut fermé, au début des années 1970, une petite scène électroacoustique riche et vivante - vraiment progressiste ou même avantgardiste - commençait à se développer en RDA. Il y avait des grands personnages comme Paul-Heinz Dittrich (1930), Georg Katzer (1953) ou Lothar Voigtländer, mentionné plus haut, qui ont fait avancer la découverte des possibilités vraiment progressistes et expérimentales d'une musique électroacoustique en suivant des chemins divers, mais guidés en tout cas par un esprit pionnier. 

déjà des termes esthétiques, pour le sujet traité ici cela n'a de sens de comprendre l'électroacoustique que comme catégorie technologique et sociale. Donc, lorsque nous mentionnons ici la musique électroacoustique, il s'agit d'une musique qui ne pourrait pas exister sans électricité, que ce soit dans sa production ou dans sa réception. L'électricité est en effet nécessaire pour la production, la manipulation ou encore la distribution des sons. La musique traditionnelle seulement enregistrée ou distribuée par haut-parleurs n'est - selon cette définition - pas électroacoustique.

Il faut ensuite souligner que la représentation esthético-historiographique du $\mathrm{xx}^{\mathrm{e}}$ siècle attribue de manière presque générale une attitude progressiste au concept de cette musique. Ceci s'explique par le fait que ce concept résulte de l'usage des nouvelles technologies, et de la nécessité de créer des arts appliqués aux nouveaux médias comme la radio. Il provient donc de la recherche de nouvelles formes de présentation et de réception. Il est vrai que les concepts radiophonique et plus tard acousmatique ont révolutionné en grande partie la réception musicale. Ce serait un autre sujet de discuter, si ce progressisme social correspond d'une manière ou d'une autre à un progressisme esthétique. Pour le moment, il est suffisant de dire qu'un certain progressisme était attribué à la tradition électroacoustique des années de la RDA. Même en gardant cela à l'esprit, certains concepts esthétiques de l'époque restent plus déterminants que d'autres. Bien sûr, la plupart des chercheurs, des musiciens, ou du public en général penserait d'abord à Stockhausen, à Kagel ou à Pousseur, à Nono ou à Henry. Cela s'explique d'abord par l'originalité et la notabilité de ces approches des nouveaux médias, mais aussi par la présence des compositeurs sur le marché mondial de l'avant-garde. L'interrelation entre ces deux éléments est déterminante aussi pour le développement des représentations musico historiques. Pour la musique moderne de l'Est, les conditions préalables sont en général différentes. 


\section{La représentation historique de la musique électroacoustique en RDA et la guerre froide}

21 Il serait donc assez simple de dire - comme il a parfois déjà été dit - que la musique électroacoustique n'existait pas en RDA, parce qu'elle était officiellement interdite du fait de son progressisme abstrait qui désobéissait à l'esthétique dictée par le régime, autrement dit au réalisme socialiste. Mais ce n'est pas le cas. Une sorte de monde parallèle se développait, se refusant de presque tout - de l'accessible comme de l'inaccessible.

Lorsque l'on parle de la musique électroacoustique sous la guerre froide, il faut souligner le fait que parler d'une guerre suppose généralement qu'il y a au moins deux combattants, représentants deux principes contraires, pour lesquels il fait sens à leurs yeux de se battre. Et cela suppose au moins une différence grave qui est visible pour tous. Cependant, l'idée même de se battre, que ce soit pour une idée, un territoire, des ressources ou d'autres choses, concrètes ou abstraites, autrement dit ce modèle de guerre - froide ou chaude, implique qu'il y ait assez de moments comparables ou communs. On se compare - cette action fait partie du processus de se battre; et on se bat sur un fond commun.

C'est justement ce principe qui fait de la musique électroacoustique de la RDA une sorte de prototype pour l'analyse des mécanismes d'échange culturels sous la guerre froide.

L'Allemagne, du fait de sa situation socio politique, était donc le champ de bataille idéal pour la guerre froide, et pas seulement à cause des frontières géographiques réelles. Deux principes contradictoires y étaient en concurrence sur fond d'une histoire, de traditions culturelles, et d'une langue communes.

Et dans la culture des arts sérieux, notamment dans la musique, il n'y avait rien d'aussi sensible, d'aussi politique que la musique électroacoustique. Une sorte de champ de bataille en miniature, reproduisant beaucoup des constellations générales. En présentant des positions assez extrêmes, parfois avec une terminologie douteuse, elle était idéale pour toute polémique de l'un combattant l'autre dans le domaine culturel.

Posons de nouveau maintenant la question de pourquoi le premier studio électronique à l'Est a pu se développer justement à ce moment-là en RDA et avec cette structure très spéciale. La réponse est déjà plus claire. Du fait que les deux Allemagnes partageaient la même tradition et la même langue, le côté Est était toujours au courant de ce qui était fait, en toute liberté, de l'autre coté. Lorsque l'on se compare et que l'on rivalise, il faut bien évidemment pouvoir se référer à ce que l'on a vu de l'autre côté - aussi d'un point de vue officiel. Un studio permet donc d'être au courant des avancées techniques de manière privilégiée.

Et bien sûr la bataille autour du studio concerne aussi un des médias, la radio, puisqu'il fallait développer la technologie radiophonique pour garder son influence sur le marché des informations. La musique électroacoustique de cette première période est donc un produit subalterne, résultant de la volonté de l'Est de garder sa position au niveau des médias et en particulier de la radio. Quand les gens au studio d'Adlershof se réfèrent à la tradition de la Rundfunkversuchsstelle de l'entre-deux-guerres à BerlinCharlottenburg, ils se définissent de manière inconsciente aussi comme héritiers d'un développement linéaire, d'une tradition humaniste. Comme ça, ils se considèrent 
comme progressistes au niveau scientifique et technologique, et en même temps comme les bons héritiers, les humanistes.

La radio a vis-à-vis de ce champ de bataille une sorte de double fonction et de double caractère : elle influence le développement de la musique électroacoustique comme institution en offrant des possibilités de production, des commandes d'œuvres, donc un cadre esthétiquement assez indépendant. Mais, notamment pour la deuxième génération de compositeurs électroacoustiques, la radio de l'Ouest joue un rôle déterminant dans le développement de la musique produite pendant cette période. Il faut souligner que même pour l'Ouest cette sorte de musique était aussi - à côté de quelques festivals - distribuée premièrement par ce medium. Et les musiciens à l'Est étaient toujours bien au courant des mouvements esthétiques à l'Ouest. Ce n'était par contre absolument pas le cas du flux d'informations circulant dans l'autre sens, mais ce serait un autre sujet.

Cependant, c'est cette radio de l'Ouest qui influence intensivement le développement de la musique électroacoustique d'une deuxième phase. La musique électroacoustique de cette phase est produite à l'Est sans accès aux studios publics, sans trop de soutien officiel et donc sans pressions extrêmes, même si le soutien officiel n'est pas complètement absent. Les séjours d'étude dans des studios à l'étranger étaient permis et même payés par l'État pour certains des compositeurs mentionnés.

Sur le plan esthétique, ce n'est donc pas du tout une protestation politique qui motive en premier lieu le mouvement électroacoustique des années 1970 en RDA comme on l'interprète parfois, mais la recherche intense de nouveaux moyens d'expression.

Le studio à Adlershof - où Siegfried Matthus, Bernd Wefelmeyer et Frederick Rzewski ont produit les seules œuvres vraiment avant-gardistes sous un regard objectif terminait la production de musique électroacoustique dans des conditions assez obscures. Finalement, c'est cette sorte de dé-officialisation, qui ouvrit la porte à une relative incertitude, mais par conséquent aussi à une attitude plus avant-gardiste. C'est le début d'une période productive pour la musique électroacoustique en RDA.

\section{Les trois phases du développement de la musique électroacoustique en RDA}

32 En nous appuyant sur le résultat de recherches effectuées dans le cadre d'un vaste projet portant sur la musique électroacoustique en RDA, nous distinguons trois phases historiques de la musique électroacoustique en RDA.

Il s'agit tout d'abord des années 1950 jusqu'au début des années 1970 - discutées jusqu'ici. Pour résumer rapidement : pendant cette période, la radio nationale fonde un studio de recherche et de création assez réputé. Des compositeurs nationaux (Matthus par exemple) ou étrangers (Rzewski par exemple) y sont invités pour y réaliser des commandes. On y développe l'instrument électronique "Subharchord » et y réalise des musiques pour le concert, la radio et la télévision. Le studio disparait ensuite pour des raisons obscures.

Il y a ensuite une période courte et marquante, déjà mentionnée également, qui a façonné la représentation virulente de la musique électroacoustique de la RDA qui opère jusqu'à aujourd'hui. Elle s'étend du milieu des années 1970 au début des années 1980. Officiellement, il n'y a pas de studios dans le pays et les compositeurs qui 
s'intéressent à la musique électroacoustique sont fortement critiqués par leurs collègues dans les associations de compositeurs. Une musique progressiste subversive se développe pourtant, souvent dans des situations paradoxales, mais avec une bonne connaissance de ce que l'on fait à l'Ouest de l'Allemagne. Des petits festivals ou encore le petit studio au théâtre "Palast der Republik" propagent malgré tout cet art et ouvrent un large podium aux musiciens pour jouer leurs propres œuvres électroacoustiques ou même des pièces de leurs collègues de l'Ouest.

On assiste enfin à une période extrêmement dynamique allant de la fin des années 1970 à la fin de l'État socialiste. Durant cette période, une scène forte, bénéficiant d'une large audience et d'une profonde résonance, se développe pour des raisons diverses. Quelques acteurs voyagent à l'Ouest, rapportant appareils et machines afin de produire de la musique électroacoustique de manière indépendante. Des liens bien établis existent avec des festivals et des studios en Allemagne de l'Ouest ainsi qu'avec d'autres pays de l'Est. Les changements politiques influencent également ce développement. En 1986 l'Académie des beaux-arts à Berlin-Est ouvre, sous la direction de Georg Katzer, un large studio de musique électronique. Il offre aussi des possibilités assez professionnelles de production, mais avant tout une sorte de podium pour un dialogue général sur les arts audio, des perspectives avant-gardistes et autres. Et parce que la musique électroacoustique était généralement vue comme quelque chose de subversif, de contraire aux positions officielles, cela attirait toujours un large public. D'une manière générale, cette période est caractérisée par des studios privés gagnant de plus en plus importance à l'Ouest également.

Sur le plan esthétique et partiellement même aussi technologique, les résultats musicaux ne sont plus tellement différents de ce qui est fait dans le reste du monde. Les théâtres, avec le besoin de musiques de scène, proposent une autre plateforme pour la composition électroacoustique.

\section{BIBLIOGRAPHIE}

BERG Michael, HOLTSTRÄTER Knut et MASSOw Albrecht von (dir.), Die unerträgliche Leichtigkeit der Kunst. Ästhetisches und politisches Handeln in der DDR, Köln, Weimar, Wien, Böhlau, 2007.

BERG Michael, MASSOW Albrecht von et NOESKE Nina (dir.), Zwischen Macht und Freiheit: Neue Musik in der DDR, Köln, Weimar, Wien, Böhlau, 2004.

BLUMRÖDER Christoph von, Neue Musik im Spannungsfeld von Krieg und Diktatur, Wien, Der Apfel, 2009.

KÄMPFER Frank et RIENÄCKER Gerd, « ... von moralischen Konnotationen befreien: über DDRMusikgeschichte vor und nach 1989/90 ", Neue Zeitschrift für Musik, nº 171/2, 2010, p. 12-15. LINDENBERGER Thomas, Massenmedien im Kalten Krieg. Akteure, Bilder, Resonanzen, Köln, Weimar, Wien, Böhlau, 2006. 
MIERSCH Manfred, «Subharmonische Mixturen mit dem Subharchord », 2003. Disponible sur <http://www.subharchord.de/index-Start.html> [consulté le 10/06/2010].

MIERSCH Manfred, « Der Kampf um die Klänge. Die Wiederentdeckung des einzigartigen elektronischen Instrumentes Subharchord », Forum Musikbibliothek. Beiträge und Informationen aus der musikbibliothekarischen Praxis, $\mathrm{n}^{\circ}$ 26/2, 2005, p. 201-208.

MüHL-BENNINGHAUS Wolfgang, « Rundfunk in der SBZ/DDR », Rundfunkpolitik in Deutschland, 2, München, dtv, 1999, p. 795-873.

NAUCK Gisela (dir.), Positionen 81, « Experiment DDR », Berlin, 2009.

OELERS Harriet, « „Vorbereitung für einen Weltkrieg mit Atomwaffen“. Die Anfänge der elektroakustischen Musik in der DDR », dans T. Böhme-Mehner, K. Mehner et M. Wolf (dir.), Elektroakustische Musik: Technologie, Ästhetik und Theorie als Herausforderung an die Musikwissenschaft / Electroacoustic Music: Technologies, Aesthetics, and Theories - a Musicological Challenge (actes du colloque de l'université de Leipzig, 20-22 septembre 2007), Essen, Die Blaue Eule, 2008, p. 132-142.

STEINKE Gerhard, «Mit den Ohren sehen - mit den Augen hören ». Ansprüche und Wege zum optimalen Hörerlebnis. Beiträge zur « Hörkunst »-Akustik und Audiotechnologie, Berlin, Kopie Druck, 2010.

STöcK Gilbert, Neue Musik in den Bezirken Halle und Magdeburg zur Zeit der DDR. Kompositionen, Politik, Institutionen, Leipzig, Schröder, 2008.

TISCHER Matthias (dir.), Musik in der DDR: Beiträge zu den Musikverhältnissen eines verschwundenen Staates, Berlin, Kuhn, 2005.

\section{RÉSUMÉS}

Nous pouvons distinguer trois phases historiques de la musique électroacoustique en RDA. Il s'agit tout d'abord des années 1950 jusqu'au début des années 1970. Pendant cette période, la radio nationale fonde un studio de recherche et de création assez réputé. Du milieu des années 1970 au début des années 1980 est créée ensuite une musique progressiste subversive, dont l'image virulente opère jusqu'à aujourd'hui. Nous traiterons enfin la période extrêmement dynamique allant de la fin des années 1970 à la fin de l'État socialiste. Durant cette période, une scène forte, bénéficiant d'une large audience et d'une profonde résonance, se développe pour des raisons diverses.

Die Entwicklung elektroakustischer Musik in der DDR lief weitgehend in drei Phasen ab. Zunächst handelt es sich dabei um die Periode der 1950er bis in die Anfänge der 1970er Jahre. Während dieser Periode gründete und betrieb das staatliche Radio ein verhältnismäßig angesehenes Forschungs- und Produktionsstudio. In der Folge sehen wir von der Mitte der 1970er Jahre bis zum Beginn der 1980er eine kurze aber markante Phase, die das gängige Bild einer progressiven und gleichzeitig subversiven elektroakustischen Musik in der DDR geprägt hat, wie es noch heute als gültig vermittelt wird. Wir finden schließlich eine extrem dynamische Periode, die vom Ende der 1970er Jahre bis zum Ende des sozialistischen Staates führt. Während dieser Zeit bildet sich aus verschiedenen Gründen eine starke Elektroakustik-Szene heraus, die vom Zuspruch eines großen Publikums und nachhaltiger Resonanz profitieren kann. 
INDEX

Schlüsselwörter : Elektroakustische Musik, DDR, Sozialistischer Realismus, Labor für akustischmusikalische Grenzprobleme, Subharchord

Mots-clés : musique électroacoustique, RDA, Subharchord, réalisme socialiste, Labor für akustisch-musikalische Grenzprobleme

\section{AUTEUR}

TATJANA BÖHME-MEHNER

Leipzig / Halle-Wittenberg (Allemagne) 